\title{
Acknowledgments
}

The editors thank Santa Clara University's Center for Applied Ethics for its generous support, and our editor at Cornell, John Ackerman, for his encouragement throughout.

M. J. M. and W. A. P. 



\section{THE CONSTITUTION OF RIGHTS}


